\title{
SYNTHESIS AND ANTIMICROBAIAL EVALUTION OF 3-ISOBUTYL-2,6-DI(THIOPHENYL-3) PIPERIDIN-4-ONE DERIVATIVES
}

\author{
Kholoud Fahed Hamak, F.Kandil, S.Hamo \\ Department of Chemistry, Faculty of Science, University of Damascus, Syria \\ Email:khlood.hamak@yahoo.com
}

\begin{abstract}
In awide a program of extensive search for new and efficient antimicrobial agents,3isobutyl -2,6-di(thiophenyl-3)piperidin-4-one(1) and its derivatives (2-4) were synthesized and characterized by IR,LC-MS, ${ }^{1} \mathrm{H}-\mathrm{NMR},{ }^{13} \mathrm{C}-\mathrm{NMR}$. The synthesized compounds 1-5 were tested for their antibacterial activity in vitro against streptococcus ,bacillus subtilis, Esherichia coli, pseudomonas, and Klebsiella peumoniae. as the standard drug" streptomycin" was used.
\end{abstract}

Keywords: 3-isobutyl-2,6-di(thiophenyl-3) piperidin-4-one ${ }^{1} H,{ }^{13} C-N M R$ spectra, Antibacterial activity, E.coli

\section{INTRODUCTION}

Piperidine heterocycles play an important role in the field of medicinal chemistry many 2,6disubstituted derivatives of this class have been found to possess useful biological activities such as antiviral ,antitumor[1,2], localanesthetic[3] , herbicidal, insecticidal, fungicidal bactericidal, antiinflammatory, anti-histaminic, hypotensive, anticancer, centeral nervous system stimulant and depressant and nerve activities and so forth $[4,5,6,7]$.

The chemistry of hydrazine derivatives such as thiosemicarbazide and its hydrazones is of immense interest owing to their wide synthetic and analytical app- lications and biological activities [8] which leads to a disruption of the pituitary-thyroid hormonal regulatory system :effective antidotes to paraquat toxicity derivative piperidine are also biologically important and act as neurokinin receptor antagonists and analgesic and anti- hypertensive agents [9]. Aiming at extending our knowledge in structure activity relationship, we considered that it is valuable to synthesize asystem which unites biolabile functional groups like thiosemicarbazide and hydroxylamine into 3-Isobutyl-2,6di(thiophen-3-yl) piperidin-4-one nucleus.

\section{EXPERIMENTAL PART}

\subsection{MATERIALS}

Thin-layer chromatography(TLC) was performed to ascertain proof of the reaction and purity of products. melting points were recorded on a Stuart instrument, IR Spectrometer were recorded on Jusco 300 FTIR Spectrometer Mass spectra were recorded on a VG 11-250 data acquisition, ${ }^{1} \mathrm{H},{ }^{13} \mathrm{C}$ NMR Spectra were recorded on Brucker $400 \mathrm{MHz}$ spectrometer .samples were prepared by dissolving $10 \mathrm{mg}$ of substance in $0.5 \mathrm{ml}$ of DMSO or $\mathrm{CDCl}_{3}$ containing $1 \%$ trimethyl silane (TMS). Unless otherwise stated, all the starting materials and reagents 
were of high grade ,purchased from Aldrich ,Fluka and Merck .All the solvents were distilled prior to use.

2.2 SyNTHESIS: SYNTHESIS OF 3-ISOBUTYL-2,6DI(THIOPHENYL-3) PIPERIDIN-4-ONE AND ITS DERIVATIVES:

\subsubsection{3-Isobutyl-2,6-di(thiophen-3-yl) piperidin-4-} one: (1)

A mixture of ammonium acetate (0.5 $\mathrm{mol})$, thiophene-2-carbaldehyde ( $1 \mathrm{~mol})$

and 5-methylhexan-2-one $(0.5 \mathrm{~mol})$ in distilled ethanol was first heated to boiling .after cooling ,the viscous liquid obtained was dissolved in ether $(200 \mathrm{ml})$ and shaken with $10 \mathrm{ml}$ of concentrated hydrochloric acid. The precipitated hydrochloride of 3-isobutyl-2,6di(thiophenyl-3) piperidin-4-one was removed by filtration and washed first with 40 $\mathrm{ml}$ mixture of ethanol and ether (1:1) and then with ether to remove most of the coloured impurities. The base was liberated from an alcoholic solution by addition of aqueous ammonia and then diluted with water . It was recrystallized from ethanol .[10]

2.2.2 3-Isobutyl-4-oxo-2, 6-di (thiophen-3-yl) piperidin-1-ium 2,4, 6-trinitroph-enolate: (2)

(0.01 mol)of 3-Isobutyl-2,6-di(thiophen-3yl)piperidin-4-one dissolved in $5 \mathrm{ml}$ of ethanol was mixed with $(0.01 \mathrm{~mol})$ of picric acid dissolved in $5 \mathrm{ml}$ of Ethanol. This mixture was shaken well. The yellow needles formed were recrystallized in benzene [11]

2.2.3 3-Isobutyl-2,6-di(thiophen-3-yl) piperidin-4one oxime: (3)

About $\quad(0.05 \mathrm{~mol})$ of $\quad 3-$ Isobutyl-2,6di(thiophen-3-yl) piperidin-4-one and sodium acetate trihydrate $(0.15 \mathrm{~mol})$ were dissolved in boiling ethanol and hydroxylamine hydrochloride $(0.06 \mathrm{~mol})$ was added. The mixture was heated stirred for 3-4 h then allowed to stand overnight, and poured into crushed ice. The separated solid was filtered off and recrystallized from ethanol .[12]

2.2.4 3-Isobutyl-2,6-di(thiophe-3-yl) piperidin-4onethiosemicarbazone: (4)

(0.01 mol)of3-Isobutyl-2,6-di(thiophen-3-yl) piperidin-4-one dissolved in boiling ethanol (20ml),2-3drops of con . $\mathrm{HCl}$ was added (excess of acid is bad to the reaction) then thiosemicarbazide $(0.34 \mathrm{~g}, 3.77 \mathrm{mmol})$ was added and the reaction mixture was refluxed for 3 hours on a water bath. The progress of reaction was monitored by TLC.After the complation of reaction, the reaction mixture was brought to room temperature and the mixture was slowly poured into ice -cold water $(400 \mathrm{ml})$ with constant stirring, the crude product was precipitated. The compound was recrystallized in hot methanol

2.2.5 3-Isobutyl-2,6-di(thiophe-3-yl) piperidin-4one semicarbazone: (5)

(0.01 mol)of 3-Isobutyl-2,6-di(thiophen-3-yl) piperidin-4-one dissolved in boiling ethanol $(20 \mathrm{ml})$. Followed by addition of semicarbazidehydrochloride $(0.34 \mathrm{~g}, 3.77 \mathrm{mmol})$. The contents were refluxed for 3 hours on a water bath .The progress of reaction was monitored by TLC .After the completion of reaction ,the contents were brought to room temperature, the separated solids were filtered.After cooling the mixture was slowly poured into ice - cold water $(400 \mathrm{ml})$ with constant stirring. The crude product was precipitated. The compound was Recrystallized in hot methanol [13]

\section{RESULTS AND DISCUSSION}

The synthesized compound are illustrated in Schem 1. 3-Isobutyl-2,6-di (thiophenyl-3) piperidin-4-one 1 was obtained by the condensation of thiophen-2- carbaldehyde, ammonium acetate and 5-methyl-hexanone-2 in a ratio of $2: 1: 1$. The piperidinim picarte 2 was prepared by mixing equimolar solution of 
piperidin-4-one 1 with picric acid in ethanol and stirring the solution for $30 \mathrm{~min}$. Isobutyl2,6-di(thiophen-3-yl)piperidin-4-one oxime3 was synthesized by treatment of 3-isobutyl2,6-di(thiophen-3-yl)

piperidin-4-one 1 with hydroxylamine hydrochloride and sodium acetate in 1:1 ratio3Isobutyl-2,6-di(thiophe-3-yl)

piperidin-4-one thiosemicarbazone 4 and 3-Isobutyl-2,6-di(thiophe-3-yl) piperidin-4one semicarbazone 5 were synthesized by Treatment of 3-isobutyl -2,6-di(thiophe-3yl)piperidin-4-one1 with thiosemicarbazide and semicarbazide respectively .

Chemical data for compounds 1-5 are provided in Table 2.

\subsection{ChARACTERIZATION COMPOUNDS}

All synthesis compounds characterization in Table 1 .

TABle 1: Spectral Data OF THE SyNTHESIZED COMPOUND

\begin{tabular}{|c|c|}
\hline $\begin{array}{c}\tilde{B} \\
\dot{0} \\
\dot{0} \\
\dot{0}\end{array}$ & Spectral characterization data \\
\hline 1 & 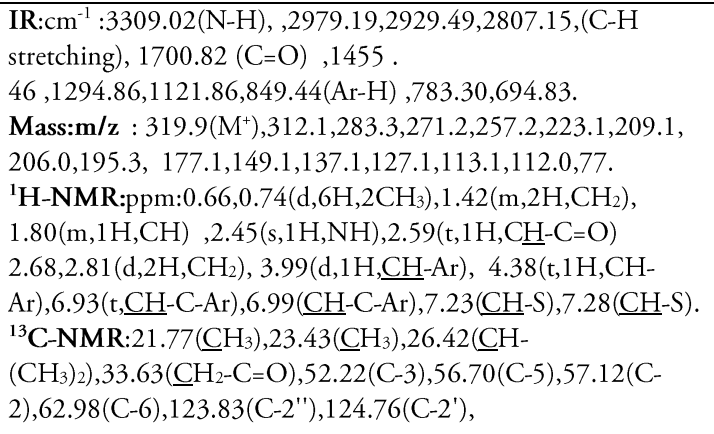 \\
\hline 2 & 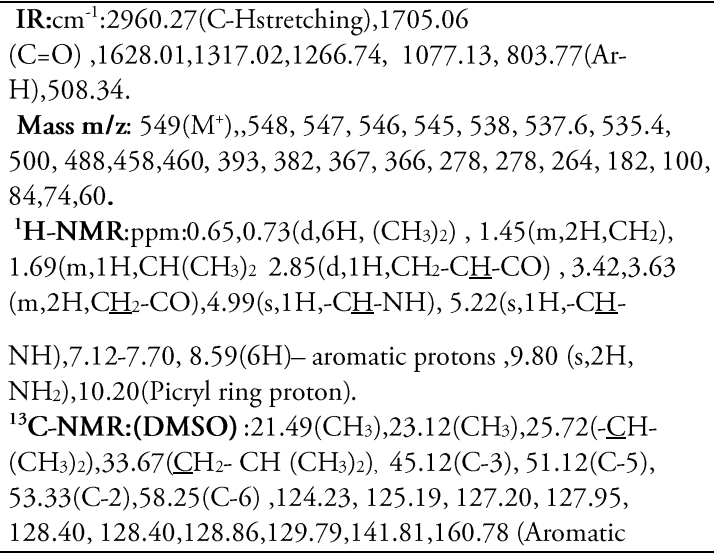 \\
\hline
\end{tabular}

carbons), 201.78(C-4).

3 IR: $\mathrm{cm}^{-1}: 3309.02(\mathrm{~N}-\mathrm{H}), 3214.54(\mathrm{OH}), 2963.62,(\mathrm{C}-\mathrm{H}$ stretching),

$1242.12,1076.14,994.77,695.75,418$.

Mass:m/z,335.1(M+1),318,317,315,311,300,269,257, 244,234, 225

,217,207,191,182,178, 163.2,153.2,100,84,74,60.

${ }^{1} \mathrm{H}-\mathrm{NMR}: \mathrm{CDCl}_{3}: 0.67,0.77\left(\mathrm{~d}, 6 \mathrm{H},\left(\mathrm{CH}_{3}\right)_{2}\right), 1.57,1.74(\mathrm{~m}$, $2 \mathrm{H}, \mathrm{CH}_{2}$ ),

$2.06\left(\mathrm{t}, 2 \mathrm{H}, \mathrm{C} \underline{\mathrm{H}}-\left(\mathrm{CH}_{3}\right)_{2}\right), 2.32\left(\mathrm{CH}_{2}-\mathrm{CH}-\right.$

$\left.\left(\mathrm{CH}_{3}\right)_{2}\right), 2.46\left(\mathrm{t}, 1 \mathrm{H}, \mathrm{N}=\mathrm{C}-\underline{\mathrm{CH}}-\mathrm{CH}_{2}\right)$

$2.50(\mathrm{~s}, 1 \mathrm{H}, \mathrm{NH}), 3.91(\mathrm{~d}, 1 \mathrm{H}, \mathrm{C} \underline{\mathrm{H}}-\mathrm{N}), 3.91(\mathrm{~d}, 1 \mathrm{H}, \mathrm{CH}$

$\mathrm{N}), 4.22(\mathrm{~s}, 1 \mathrm{H}, \mathrm{N}-\mathrm{O} \underline{\mathrm{H}}), 6.92-$

8.31(Aromatic protons)

${ }^{13}$ C-NMR: $\mathrm{CDCl}_{3}: 21.75\left(\mathrm{CH}_{3}\right), 23.77\left(\mathrm{CH}_{3}\right), 25.72(-$

$\left.\underline{\mathrm{CH}}(\mathrm{CH} 3)_{2}\right), 34.99\left(\mathrm{CH}_{2}-\mathrm{CH}\left(\mathrm{CH}_{3}\right)_{2}\right), 35.06(\mathrm{C}-3), 48.22(\mathrm{C}-$

5) $, 56.33(\mathrm{C} 2), 63.84(\mathrm{C} 6), 123.77,124.39,125.08,125.68,126.16$, 126.56:aromatic carbons, $145.95\left(\mathrm{C}-1^{\prime}\right), 147.24\left(\mathrm{C}-1^{\prime \prime}\right), 158.74(\mathrm{C}-$ 4).

$4 \quad$ IR: $\mathrm{cm}^{-1} 3420.51\left(\mathrm{NH}_{2}\right), 3369.48\left(\mathrm{NH}_{2}\right), 3245.73,3148.64$ ,2949.25,(C-stretching) ,1483.46(C=S),1386.17,1114.14(N-C N).,464.44.

Mass:m/z 392(M+),378,376.1,355.1 ,338.3, 329.1 ,321, 315, $300,297,282,266,265,209,206,193,182,167,163,154.2,84.2$.

${ }^{1} \mathrm{H}-\mathrm{NMR}: \mathrm{CDCl}_{3}: 0.61,0.73\left(\mathrm{~d}, 6 \mathrm{H},\left(\mathrm{CH}_{3}\right)_{2}\right), 1.70,1.86(\mathrm{~m}$,

$\left.2 \mathrm{H}, \mathrm{CH}_{2}\right)$,

$=2.11\left(\mathrm{~m}, 2 \mathrm{H}, \mathrm{CH}-\left(\mathrm{CH}_{3}\right)_{2}\right), 2.36\left(\mathrm{CH}_{2}-\mathrm{CH}-\right.$

$\left.\left(\mathrm{CH}_{3}\right)_{2}\right), 2.49\left(\mathrm{t}, 1 \mathrm{H}, \mathrm{N}=\mathrm{C}-\underline{\mathrm{CH}}-\mathrm{CH}_{2}\right)$

$3.87(\mathrm{~d}, 1 \mathrm{H}, \mathrm{C} \underline{\mathrm{H}}-\mathrm{N}), 4.36(\mathrm{~d}, 1 \mathrm{H}, \mathrm{C} \underline{\mathrm{H}}-\mathrm{N}), 6.98(\mathrm{~s}, 1 \mathrm{H}, \mathrm{NH}), 6.99$

8.21 (Aromatic protons), $10.16\left(\mathrm{~s}, 2 \mathrm{H}, \mathrm{NH}_{2}\right)$

${ }^{13} \mathrm{C}-\mathrm{NMR} \mathrm{CDCl} 3: 22.33\left(\mathrm{CH}_{3}\right), 24.24\left(\mathrm{CH}_{3}\right), 26.45 \mathrm{CH}\left(\mathrm{CH}_{3}\right)_{2}$, $35.33\left(\mathrm{CH}_{2}-\mathrm{CH}\left(\mathrm{CH}_{3}\right)_{2}=38.09(\mathrm{C}-3), 50.63(\mathrm{C}-5), 56.63(\mathrm{C}-2)\right.$, 63.51(C-6) ,124.29,124.84125.21,125.84,

126.12,126.98,146.49,148.21,(Aromaticcarbons), 154.66(C4) $179.73(\mathrm{C}=\mathrm{S})$

$5 \quad$ IR $\mathrm{cm}^{1-}: 3465.61\left(\mathrm{NH}_{2}\right), 3183.42(\mathrm{NH}), 2952.34,2867.21(\mathrm{C}-\mathrm{H}$ stretching),1693.30,1574.32(C=O),1434.05,696.07,596.23. Mass :m/z :376(M+),375.1,372.9,367.0,358.9,358.1,357.2,350. 8,349.7,345.7,341.9,336.1,334.0,332.9,331.8, 300,297,282,266, 265, 209,206,193,182,167,163,154.2,84.2. ${ }^{1} \mathrm{H}-\mathrm{NMR}: \mathrm{CDCl}_{3}$ : 0.64,0.82(d,6H, $\left.\left(\mathrm{CH}_{3}\right)_{2}\right), 1.72,1.85(\mathrm{~m}$, $\left.2 \mathrm{H}, \mathrm{CH}_{2}\right), 2.28\left(\mathrm{~m}, 2 \mathrm{H}, \mathrm{CH}-\left(\mathrm{CH}_{3}\right)_{2}\right), 2.45\left(\mathrm{CH}_{2}-\mathrm{CH}-\right.$

$\left.\left(\mathrm{CH}_{3}\right)_{2}\right), 2.49(\mathrm{t}, 1 \mathrm{H}, \mathrm{N}=\mathrm{C}-\underline{\mathrm{CH}}-$

$\left.\mathrm{CH}_{2}\right), 4.20(\mathrm{~s}, 1 \mathrm{H}, \mathrm{NH}), 4.39(\mathrm{~d}, 1 \mathrm{H}, \mathrm{CH}-\mathrm{N}), 6.03$

(s,2H,NH ), 6.96(s,1H,NH), (6.99-8.46(Aromatic protons),

${ }^{13} \mathrm{CNMR}: \mathrm{CDCl}_{3}: 21.64\left(\mathrm{CH}_{3}\right), 23.34\left(\mathrm{CH}_{3}\right), 26.30 \underline{\mathrm{CH}}\left(\mathrm{CH}_{3}\right)_{2}, 35$. $25\left(\mathrm{CH}_{2}-\mathrm{CH}\left(\mathrm{CH}_{3}\right)_{2}\right.$

37.11(C-3),49.67 (C-5) ,56.11(C-2),62.86(C-6) ,123.75,124.46 $125.06,126.14,126.58,146.18,146.56,150.33(\mathrm{C}=\mathrm{O})$, $158.04(\mathrm{C}=\mathrm{N})$

TABLE 2: CHEMICAL DATA FOR COMPOUNDS (1-5)

\begin{tabular}{|l|l|l|l|l|}
\hline Compound & $\mathrm{R}_{\mathrm{f}}$ & $\mathrm{m} \cdot \mathrm{p}^{0} \mathrm{C}$ & $\begin{array}{l}\text { Molecular } \\
\text { formula }\end{array}$ & Yield\% \\
\hline 1 & 0.95 & 120 & $\mathrm{C}_{17} \mathrm{H}_{21} \mathrm{NOS}_{2}$ & $80.02 \%$ \\
\hline 2 & 0.86 & 185 & $\mathrm{C}_{23} \mathrm{H}_{24} \mathrm{~N}_{4} \mathrm{O}_{8} \mathrm{~S}_{2}$ & $70.23 \%$ \\
\hline 3 & 0.52 & 163 & $\mathrm{C}_{17} \mathrm{H}_{24} \mathrm{~N}_{4} \mathrm{O}_{8} \mathrm{~S}_{2}$ & $69.45 \%$ \\
\hline 4 & 0.96 & 202 & $\mathrm{C}_{18} \mathrm{H}_{24} \mathrm{~N}_{4} \mathrm{~S}_{3}$ & $63.45 \%$ \\
\hline 5 & 0.75 & 195 & $\mathrm{C}_{18} \mathrm{H}_{24} \mathrm{~N}_{4} \mathrm{OS}_{2}$ & $45.23 \%$ \\
\hline
\end{tabular}




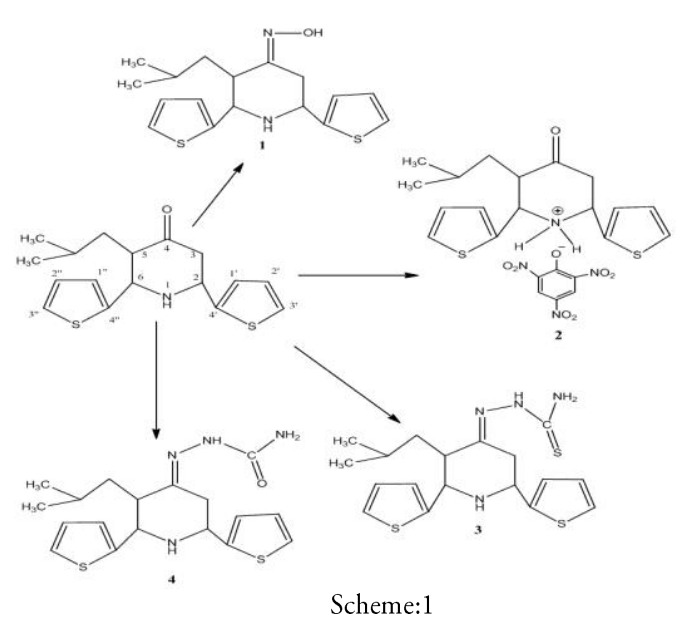

3.2.Biological Activity

The synthesised compound (1-5) were tested for their antimicrobial activity in vitro against four speices of bacteria (klebsiella‘Escherichiacoli، Staphylococcus aureus ' Salmonella typhi) using filter paper disc method The screened compounds were dissolved individually in DMSO(dimethyl sulfoxide) in order to make up a solution of 1500-1000-500 PPm concentration for each of these compounds. Filter paper discs(WhitmanNo.1filter paper, $5 \mathrm{~mm}$ diameter) were saturated with the solution of these compounds. The discs were placed on the surface of solidified Nutrient agar dishes seeded by the tested bacteria. The diameters of inhibition zones $(\mathrm{mm})$ were measured at the end of an incubation period, which was $24 \mathrm{~h}$ at $37 \mathrm{C}$ for bacteria. Discs saturated with DMSO are used as solvent control. streptomycin $25 \mathrm{ppm}$ was used as reference substance for bacteria. Streptomycin was the first antibiotic used against Mycobacterium tuberculosis and it was used for years in monotherapy regimens ,leading to the emergence of resistance. Besides its other antibiotic properties, it is one of the most widely used agents for dermatological reasons .In general ,all the synthesized biolabile compounds 1-5 exhibited a wide range of modest antibacterial activity especially against gram negative bacteria $[14,15,16]$. Tables 3 and 4.

TABLE 3: EFFECT OF THE COMPOUND $(1,2)$ ON THE GROWTH OF BACTERIA (ZONE OF INHIBITION IN MM)

\begin{tabular}{|c|c|c|c|c|c|c|}
\hline \multirow{2}{*}{$\begin{array}{c}\text { Micro } \\
\text { organisms }\end{array}$} & \multicolumn{3}{|c|}{ Compound3 } & \multicolumn{3}{c|}{ Compound 4 } \\
\cline { 2 - 7 } & 500 & 1000 & 1500 & 500 & 1000 & 1500 \\
$\mathrm{ppm}$ & $\mathrm{ppm}$ & $\mathrm{ppm}$ & $\mathrm{ppm}$ & $\mathrm{ppm}$ & $\mathrm{ppm}$ \\
\hline $\begin{array}{c}\text { Staphylococo } \\
\text { us aureus }\end{array}$ & + & ++ & +++ & - & + & ++ \\
\hline $\begin{array}{c}\text { Salmonella } \\
\text { typhi }\end{array}$ & + & + & ++ & + & ++ & ++ \\
\hline $\begin{array}{c}\text { Esherichia } \\
\text { coli }\end{array}$ & + & ++ & +++ & +++ & ++ & +++ \\
\hline Klebsiella & + & + & ++ & + & ++ & +++ \\
\hline
\end{tabular}

(-)inactive, $(+)$ weaklyactive $(8-10),(+,+)$ moderatelyactive (11-12), $(+,+,+)$ strong active (13-14)

TABLE 4: EFFECT OF COMPOUND(3,4) ON THE GROWTH OF BACTERIA (ZONE OF INHIBITION IN MM)

\begin{tabular}{|c|c|c|c|c|c|c|}
\hline \multirow{2}{*}{$\begin{array}{c}\text { Micro } \\
\text { organisms }\end{array}$} & \multicolumn{3}{|c|}{ Compound1 } & \multicolumn{3}{c|}{ Compound 2} \\
\cline { 2 - 7 } & $\begin{array}{c}500 \\
\mathrm{ppm}\end{array}$ & $\begin{array}{c}1000 \\
\mathrm{ppm}\end{array}$ & $\begin{array}{c}1500 \\
\mathrm{ppm}\end{array}$ & $\begin{array}{c}500 \\
\mathrm{ppm}\end{array}$ & $\begin{array}{c}1000 \\
\mathrm{ppm}\end{array}$ & $\begin{array}{c}1500 \\
\mathrm{ppm}\end{array}$ \\
\hline $\begin{array}{c}\text { Staphylococ } \\
\text { cus aureus }\end{array}$ & - & + & ++ & - & + & ++ \\
\hline $\begin{array}{c}\text { Salmonella } \\
\text { typhi }\end{array}$ & + & + & ++ & - & ++ & +++ \\
\hline $\begin{array}{c}\text { Esherichia } \\
\text { coli }\end{array}$ & + & ++ & +++ & +++ & ++ & +++ \\
\hline Klebsiella & + & ++ & +++ & + & +++ & +++ \\
\hline
\end{tabular}

(-)inactive, $(+)$ weaklyactive (8-10),(+,+) moderatelyactive (11-12), $(+,+,+)$,strong active(13-14)

\section{CONCLUSION}

Several substituted 3-Isobutyl-2,6-di (thiophen-3-yl) piperidin-4-one (1) and its derivatives 2-5 were synthesized .compounds 1-5 were prepared, starting with thiophene-2carbaldehyde, 5-methylhexan-2-one ketone, and ammonium acetate, via the mannich reaction. Compounds 2and 3 were synthesized via protonation and oximation .compound 4,5 were synthesized 
with condensation with thiosemicarbazide and semecarbazide. ${ }^{1} \mathrm{H},{ }^{13} \mathrm{C}-\mathrm{NMR}$ Analysis show that this compounds were synthesis and this compound show good activity against grame positive bacteria more than grame negative bacteria.

\section{REFERENCES:}

[1] Macchia B.,Macchia M.,Martielli A.et al.Ibid-32-, 1997, p 231.

[2] SuleymanH., GulH.I., Asoglu, M. Pharm . Res. 47-,2003, p 471.

[3] Koji H. ,Takeshi K.,Takeru W .U.S. Pat. Appl. Publ, 2002.

[4] GanellinCR,Spickett Rgw. Compounds Affecting the central nervous system.I.4Piperidones and related compounds.J Med Chem. Vol. 8(5), 1965, p 619.

[5] GeorgivV,Petkoval B, Pharmacological studies of some central effects of tempidon. Act physiol pharmacol Bulg,. Vol.2, 1974, p 76.

[6] Nalini, N,(1997) M Pharm Dissertation ,the Tamil Nadu Dr MGR Medical University ,India Ojha V,Singh J,Bhakuni DS,Singh S, Chatterjee RK Antiparasitic agents, ppara XIV -sythesis of 5-substituted 2-carbarkoxyamino- 1H-benzimidazole as possible macrofilaricidal agents .Indian J Chem., Vol.30B, 1991, p 324.

[7] Vankov $S$,Effect of Tempidone on the cardiovascular system of experimental animals .J Nachnoizsled Khim Farm Inst. Vol.9, 1974, p 231.

[8] YadavV.K.,SenthilG.,SinghL.,ParvesM.J.Org.Che m. Vol.69, 2004, p 8131.

[9] Jayabharathi J .,Manimekalai A .,Consalata Vani T .Eur. J. Med. Chem ,2007.

[10] NollerC.R., Baliah V., J.Am. Chem. Soc., Vol.70, 1948, pp 3853-3855.

[11] Krishna kumar R,Krishnaoillay M .Indian J Hetrocyclic Chem, Vol.2,1993, p 379.

[12] Ramalingan C, Park, Y, T, Kabila, S , Synthesis, Stereochemistry, and antimicrobial evalution of Substituted piperidin-4-one oxime ethers, p European Journal of Medicinal Chemistry, 2006, pp 683-396.

[13] Aridoss G, Amirthaganesan S, Kim M ,S , Kim .J.T ,Synthesis ,Spectral and biological evalution of some new thiazolidinones and thiazoles based on t-3-alkyl-r-2,c-6diarylpiperidin-4-ones ,European Journal of Medicinal Chemistry, 2009.

[14] Clark, N.C.Cooksey, R.C.Hill, B.C. Swenson, J.M, p Tenover. F. C. Antimicrob Agents Chemother, Vol. 37, 1993, p 2311.

[15] P. Ruiz, F. Rodriguezcano, F. J. Zerolo, M. Casal, Microbial Drug Resistance Vol.8, 2002,pp 147149.

[16] Ruiz P, Rodriguez-Caon F, Zerolo FJ, Casal M, Investigation of the in vitro activity of streptomycin against Mycobacterium tuberculosis. Microb Drug Resist Vol.8(2) ,2009, p 147. 


\section{اصطناع ودراسة الفعالية الحيوية لمشتقات 3- ايزوبوتيل -6,2- ثنائي (تيوفنيل -3) بيبيريدون - 4}

$$
\text { خلود فهد حمك ، فاروق قنديل ، سامح حمو }
$$

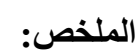

$$
\begin{aligned}
& \text { ضمن إطار برنامج البحث عن الجديد في مجال المركبات المضادة للبكتيريا وللفطور تم في هذا البحث اصطناع مركب } \\
& \text { ايزوبوتيل - 6,2- ثنائي (تيوذذفنيل -3) بيبيريدون - } 4 \text { و مشتقاته الأربع ووصفت هذه المركبات بالطرق } \\
& \text { المطيافية : IR,MASS, }{ }^{1} \mathrm{H}-\mathrm{NMR},{ }^{13} \mathrm{C}-\mathrm{NMR} \\
& \text { اختبرت فعالية المركبات المصنعة ضد البكتيريا }
\end{aligned}
$$

Klebsiella peumoniae, Esherichia coli, Staphylococcus aureus ، Salmonella typhi 